\title{
Full-Scan Accurate Mass Selectivity of Ultra-Performance Liquid Chromatography Combined with Time-of-Flight and Orbitrap Mass Spectrometry in Hormone and Veterinary Drug Residue Analysis
}

\author{
E. van der Heeft, ${ }^{a}$ Y. J. C. Bolck, ${ }^{\text {a B. Beumer, }}$, A. W. J. M. Nijrolder, ${ }^{\text {a }}$ \\ A. A. M. Stolker, and M. W. F. Nielen ${ }^{\mathrm{a}, \mathrm{b}}$ \\ ${ }^{a}$ RIKILT-Institute of Food Safety, Wageningen, The Netherlands \\ ${ }^{\mathrm{b}}$ Wageningen University, Laboratory of Organic Chemistry, Wageningen, The Netherlands
}

\begin{abstract}
The applicability of ultra-performance liquid chromatography (UPLC) combined with fullscan accurate mass time-of-flight (TOF) and Orbitrap mass spectrometry (MS) to the analysis of hormone and veterinary drug residues was evaluated. Extracts from blank bovine hair were fortified with 14 steroid esters. UPLC-Orbitrap MS performed at a resolving power of 60,000 (FWHM) enabled the detection and accurate mass measurement $(<3 \mathrm{ppm}$ error) of all 14 steroid esters at low ng/g concentration level, despite the complex matrix background. A 5 ppm mass tolerance window proved to be essential to generate highly selective reconstructed ion chromatograms (RICs) having reduced background from the hair matrix. UPLC-Orbitrap MS at a lower resolving power of 7500 and UPLC-TOFMS at mass resolving power 10,000 failed both to detect all of the steroid esters in hair extracts owing to the inability to mass resolve analyte ions from co-eluting isobaric matrix compounds. In a second application, animal feed extracts were fortified with coccidiostats drugs at levels ranging from 240 to $1900 \mathrm{ng} / \mathrm{g}$. UPLC-Orbitrap MS conducted at a resolving power of 7500 and 60,000 and UPLCTOFMS detected all of the analytes at the lowest investigated level. Thanks to the higher analyte-to-matrix background ratio, the utilization of very narrow mass tolerance windows in the RIC was not required. This study demonstrates that even when the targeted sample preparation from conventional LC-MS/MS is applied to UPLC with full-scan accurate mass MS, false compliant (false negative) results can be obtained when the mass resolving power of the MS is insufficient to separate analyte ions from isobaric co-eluting sample matrix ions. The current trend towards more generic and less selective sample preparation is expected to aggravate this issue further. (J Am Soc Mass Spectrom 2009, 20, 451-463) (c) 2009 Published by Elsevier Inc. on behalf of American Society for Mass Spectrometry
\end{abstract}

$\mathrm{T}$ To maintain sample throughput and cost-effectiveness, it will be necessary to develop generic liquid chromatography-mass spectrometry (LC-MS) screening methods for the simultaneous detection and identification of a wide range of banned and novel hormones, registered veterinary drug residues, and their metabolites. The majority of current LC-MS based hormone and veterinary drug residue analyses relies on the high sensitivity and selectivity of triple quadrupole tandem mass spectrometry (QqQ-MS/MS) systems operated in the multiple reaction-monitoring (MRM) mode. The two-stage mass selection provides the selectivity and sensitivity to enable the detection, identification, and quantification of preselected targets at low

Address reprint requests to Ing E. van der Heeft, RIKILT Institute of Food Safety, Bornsesteeg 45, P.O. Box 230, 6700 AE Wageningen, The Netherlands. E-mail: ed.vanderheeft@wur.nl URL: http://www.rikilt.wur.nl $\mathrm{ng} / \mathrm{g}$ level in complex biological matrices such as urine, feces, tissue, feed, and hair [1]. The recent introduction of ultra-performance (UP)LC and fast-switching QqQMS/MS instruments significantly increased the number of targets that can be detected in one analysis [2]. However, analytes might move out from the acquired MRM time windows. Another major inherent limitation of targeted LC-MS/MS approaches is the inability to detect novel residues such as illegally administered anabolic steroids designed to escape from veterinary control.

Full-scan MS approaches offer the advantage of the analysis of a virtually unlimited number of analytes simultaneously. Furthermore, the retrospective "posttargeted" evaluation of old data for the detection of non-“a priori"selected compounds by reconstructing any desired reconstructed ion chromatogram is an advantage of the non-targeted LC-MS approach. To
(C) 2009 Published by Elsevier Inc. on behalf of American Society for Mass Spectrometry. $1044-0305 / 09 / \$ 32.00$

doi:10.1016/j.jasms.2008.11.002
Published online November 17, 2008 Received May 30, 2008 Revised November 3, 2008 Accepted November 4, 2008 
enable the detection of residues at the required low $\mathrm{ng} / \mathrm{g}$ level, it is necessary to use very sensitive full-scan mass analyzers. Another prerequisite for a generic screening method is the development and application of less selective sample preparation procedures, thereby increasing the risk of spectral interferences and ion suppression. Thus, a key question is whether the medium mass resolving power provided by time-of-flight (TOF) instruments is sufficient to enable the detection and accurate mass measurement of residues at low $\mathrm{ng} / \mathrm{g}$ level in complex sample matrices. The utility of full-scan LC-TOFMS for the comprehensive screening of urine samples for drugs [3,4] and doping agents [5], crops for pesticide residues [6], and confirmation of antibiotics and fungicides in salmon [7] was demonstrated. Comparison of theoretical and measured isotopic patterns might be a useful additional tool to accurate mass determination and facilitates the identification process [8]. The use of high resolving power mass spectrometers, such as a linear ion trap coupled with an Orbitrap [9, 10], might further improve the confidence in screening results obtained by full-scan accurate mass LC-MS. A doping control screening method was recently developed for the analysis of doping agents in urine [11] by using LC-Orbitrap MS at 60,000 FWHM (full width at half maximum) resolving power. A recent study [12] demonstrated that an Orbitrap mass resolving power of 25,000 to 40,000 was essential to obtain sufficient selectivity to enable the detection and quantification of $N$-nitrosamines in drinking water and wastewater samples at low ng/L level. The larger dynamic range over which accurate masses can be determined of LTQ Orbitrap MS could be a distinct advantage over TOFMS [13].

The use of reconstructed ion chromatograms (RICs) with very narrow mass tolerance windows $(<10 \mathrm{ppm})$ is an efficient way to improve the selectivity of an LC-MS method. However, the use of narrow mass tolerance windows is only feasible when the mass spectrometer provides sufficient resolving power to discriminate analytes from isobaric co-eluting sample matrix compounds. It was predicted and demonstrated that a medium mass resolving power (Q)TOFMS(/MS) instrument might be critical in this respect [14]. The European Union (EU) recognizes the added value of high mass resolving power instruments for the identification of residues [15]. According to Decision 2002/ $657 / \mathrm{EC}$, a system of identification points is to be used for the confirmation of non-compliant screening results. Ions acquired with high resolving power instruments earn two identification points compared to one point for low resolving power MS. However, to qualify for high resolving power MS, the mass resolving power shall be greater than 10,000 for the entire mass range at 10\% valley definition $(\sim 20,000 \mathrm{FWHM})$. Key questions are whether full-scan high mass accuracy and medium to high mass resolving power MS instruments can compensate for the loss in selectivity versus established LC-MS/MS methods and whether the mass resolving power requirements in 2002/657/EC are adequate for demanding hormone and veterinary drug residue applications.

Studies discussing the impact of mass resolving power on the accuracy of mass measurement of residues in the presence of a complex matrix background are rare. Calbiani et al. [16] investigated the influence of spectrum processing parameters on mass measurement accuracy in the case of poor resolving power between analyte ion signals and interfering compounds. The authors showed that processing parameters of mass spectra should be carefully selected in the case of partially resolved peaks to prevent mass measurement inaccuracy. Kaufmann et al. [17] concluded that isobaric interferences are rather uncommon when using a TOFMS at mass resolving power of 5000 to 10,000 . However, they investigated the occurrence of false compliant (false negative) results only by injecting diluted binary mixtures of isobaric model substances at various relative abundances.

In this study, the performance and applicability of UPLC-TOFMS at 10,000 mass resolving power and UPLC-LTQ Orbitrap MS at 7500 and 60,000 mass resolving power were evaluated by using two demanding real-life applications: (1) steroid ester hormone residues in hair at the low $\mathrm{ng} / \mathrm{g}$ level and (2) coccidiostat drugs in animal feed at 0.2 to $2 \mu \mathrm{g} / \mathrm{g}$ level. More information about these applications are in the Supplemental section, which can be found in the electronic version of this article.

\section{Experimental}

\section{Chemicals}

Water LC/MS grade, acetonitrile LC/MS grade, and methanol HPLC supra-gradient grade were purchased from Biosolve (Valkenswaard, The Netherlands). Formic acid, p.a., was obtained from Merck (Darmstadt, Germany). Ammonium formate, 97\%, and tris(2carboxyethyl)phosphine hydrochloride (TCEP) were from Sigma-Aldrich (St. Louis, MO). Boldenone undecylenate, estradiol 3-benzoate, testosterone acetate, testosterone benzoate, testosterone cypionate, and testosterone propionate were from Sigma-Aldrich. Estradiol 17-enanthate, estradiol 17-valerate, nortestosterone phenylpropionate, testosterone decanoate, testosterone enanthate, testosterone isocaproate, testosterone phenylpropionate, and testosterone undecanoate were purchased from Steraloids (Newport, RI). Amprolium, carbadox, dimetridazole, furazolidone, ronidazole, ethopabate, and tylosin were purchased from SigmaAldrich. Meticlorpindol, olaquindox, and robenidine were obtained from Riedel de Haën (Seelze, Germany).

Individual stock solutions of steroid esters were prepared by dissolving $10 \mathrm{mg}$ of each compound in 10 $\mathrm{mL}$ methanol. Composite working standard solutions were prepared by mixing of stock solutions and diluting with water/acetonitrile/methanol (30:35:35 vol/ 
vol/vol). Calibration standard solutions of steroid esters were prepared by dilution of working standard solutions in water/acetonitrile/methanol (30:35:35 vol/ vol/vol). Concentration levels of analytes in calibration standard solutions were equal to those in fortified extracts of hair. Individual stock solutions of coccidiostats were prepared by dissolving $10 \mathrm{mg}$ of dimetridazole, ethopabate, and ronidazole in $10 \mathrm{~mL}$ methanol and $10 \mathrm{mg}$ of the other compounds in $100 \mathrm{~mL}$ methanol. Composite working standard solutions were prepared by mixing of stock solutions and diluting with methanol.

\section{Preparation of Hair Samples}

About $3 \mathrm{~g}$ of blank bovine hair samples were weighed in a funnel and washed with $\sim 300 \mathrm{~mL}$ water. After drying at $45^{\circ} \mathrm{C}$ for $24 \mathrm{~h}$, hair samples were cut into pieces of about $1 \mathrm{~cm}$. Approximately $1 \mathrm{~g}$ of hair samples were placed in a ball mill and pulverized by milling at $25 \mathrm{~Hz}$ for $4 \mathrm{~min}$. Next, $200 \pm 2 \mathrm{mg}$ of pulverized hair was weighed in a plastic tube. Two $\mathrm{mL}$ of $25 \mathrm{mM}$ TCEP solution in $0.5 \mathrm{M} \mathrm{NaH}_{2} \mathrm{PO}_{4}$ were added to the sample, the tube was placed in a head-over-head apparatus, and shaken for $1 \mathrm{~h}$. Then, $5 \mathrm{~mL}$ of methanol were added to the tube, followed by centrifugation at $1700 \mathrm{~g}$ for $5 \mathrm{~min}$. The supernatant was transferred to a plastic tube, followed by the addition of $4 \mathrm{~mL}$ water. Then the extract was applied to a Bond Elut (Varian, Palo Alto, CA) SPE cartridge containing $100 \mathrm{mg}$ of LRC-C18, previously conditioned with $2 \mathrm{~mL}$ of acetonitrile, $2 \mathrm{~mL}$ of methanol, and $2 \mathrm{~mL}$ of water. After washing with 2 $\mathrm{mL}$ of water, the SPE column was eluted with $2 \mathrm{~mL}$ of acetonitrile, followed by $2 \mathrm{~mL}$ of ethyl acetate. The eluate was collected in a plastic tube and evaporated to dryness under a stream of nitrogen with the sample at $40{ }^{\circ} \mathrm{C}$. The residue was reconstituted in $100 \mu \mathrm{L}$ of water/acetonitrile/methanol (30:35:35 vol/vol/vol). At this point the blank hair extracts were spiked with steroid esters at four different concentration levels by addition of $100 \mu \mathrm{L}$ of one of the working standard solutions; thus analyte recovery of the sample preparation was not a variable in this study and fixed at $100 \%$. The exact composition of the spiked sample extracts is given in Table 1 and correspond to $2.5-550 \mathrm{ng} / \mathrm{g}$ of hair when $100 \%$ recovery would be valid.

Hair samples were also analyzed by using MRM with a triple quadrupole instrument. An identical sample preparation procedure was used, with the exception that samples were spiked before conducting the extraction and SPE clean-up. Blank bovine hair samples were spiked by the addition of standard solutions of steroid esters to $200 \mathrm{mg}$ of pulverized hair at the concentration levels shown in Table 1.

Assuming a $100 \%$ recovery, the concentration of analytes in fortified extracts correspond to the following concentration levels in hair:

Level A: 2.5-55 ng/g

Level B: 5-110 ng/g

Level C: $10-220 \mathrm{ng} / \mathrm{g}$

Level D: 25-550 ng/g

\section{Preparation of Feed Samples}

Blank premix feed samples were milled, sieved by using a $1 \mathrm{~mm}$ sieve, and homogenized. Next, $2 \pm 0.05 \mathrm{~g}$ of homogenized sample was weighed in a plastic tube, followed by addition of $4 \mathrm{~mL}$ of $0.02 \mathrm{M} \mathrm{NaOH}$ in methanol/acetonitrile (50:50). After vigorously shaking, the tube was placed in an ultrasonic bath for $5 \mathrm{~min}$, followed by centrifugation at $1700 \mathrm{~g}$ for $5 \mathrm{~min}$. Finally, $100 \mu \mathrm{L}$ of the supernatant were transferred to a vial, and the extract was diluted with $700 \mu \mathrm{L}$ water. Diluted extracts were spiked with coccidiostats by addition of 3 to $12 \mu \mathrm{L}$ of one of the working standard solutions to the extracts, resulting in concentration levels of 15, 30, 60, and $120 \mathrm{ng} / \mathrm{mL}$, being equivalent to $240,480,960$, and $1920 \mathrm{ng} / \mathrm{g}$ of feed if the recovery were $100 \%$.

Table 1. Concentration of steroid esters in fortified extracts of bovine hair

\begin{tabular}{|c|c|c|c|c|c|}
\hline Compound & $\begin{array}{c}\text { Chemical formula } \\
{[\mathrm{M}+\mathrm{H}]^{+} \text {ion }}\end{array}$ & & & & \\
\hline Concentration & & Level A & Level B & Level C & Level D \\
\hline Boldenone undecylenate & $\mathrm{C}_{30} \mathrm{H}_{45} \mathrm{O}_{3}$ & 2.5 & 5 & 10 & 25 \\
\hline Estradiol benzoate & $\mathrm{C}_{25} \mathrm{H}_{29} \mathrm{O}_{3}$ & 2.5 & 5 & 10 & 25 \\
\hline Estradiol enanthate & $\mathrm{C}_{18} \mathrm{H}_{23} \mathrm{O}$ & 55 & 110 & 220 & 550 \\
\hline Estradiol valerate & $\mathrm{C}_{18} \mathrm{H}_{23} \mathrm{O}$ & 55 & 110 & 220 & 550 \\
\hline Nortestosterone phenylpropionate & $\mathrm{C}_{27} \mathrm{H}_{35} \mathrm{O}_{3}$ & 2.5 & 5 & 10 & 25 \\
\hline Testosterone acetate & $\mathrm{C}_{21} \mathrm{H}_{31} \mathrm{O}_{3}$ & 5 & 10 & 20 & 50 \\
\hline Testosterone benzoate & $\mathrm{C}_{26} \mathrm{H}_{33} \mathrm{O}_{3}$ & 5 & 10 & 20 & 50 \\
\hline Testosterone cypionate & $\mathrm{C}_{27} \mathrm{H}_{41} \mathrm{O}_{3}$ & 10 & 20 & 40 & 100 \\
\hline Testosterone decanoate & $\mathrm{C}_{29} \mathrm{H}_{47} \mathrm{O}_{3}$ & 10 & 20 & 40 & 100 \\
\hline Testosterone enanthate & $\mathrm{C}_{26} \mathrm{H}_{41} \mathrm{O}_{3}$ & 10 & 20 & 40 & 100 \\
\hline Testosterone isocaproate & $\mathrm{C}_{25} \mathrm{H}_{39} \mathrm{O}_{3}$ & 5 & 10 & 20 & 50 \\
\hline Testosterone phenylpropionate & $\mathrm{C}_{28} \mathrm{H}_{37} \mathrm{O}_{3}$ & 7.5 & 15 & 30 & 75 \\
\hline Testosterone propionate & $\mathrm{C}_{22} \mathrm{H}_{33} \mathrm{O}_{3}$ & 5 & 10 & 20 & 50 \\
\hline Testosterone undecanoate & $\mathrm{C}_{30} \mathrm{H}_{49} \mathrm{O}_{3}$ & 50 & 100 & 200 & 500 \\
\hline
\end{tabular}

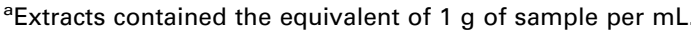




\section{TOFMS and LTQ Orbitrap MS Infusion Experiments}

Infusion experiments were performed by using a Waters (Manchester, UK) model LCT Premier orthogonal acceleration TOFMS and a ThermoFisher Scientific (Bremen, Germany) model LTQ Orbitrap XL MS. Standard solutions of testosterone acetate, estradiol benzoate, and tylosin at $1.0 \mathrm{ng} / \mu \mathrm{L}$ level were prepared by dilution of stock solutions in water/methanol (50:50 vol/vol) containing $0.1 \%$ formic acid and introduced into the ESI source of the MS at a flow rate of $3 \mu \mathrm{L} / \mathrm{min}$ by using a Harvard Apparatus (Holliston, MA) model PHD 2000 syringe pump. The TOFMS was equipped with a LockSpray dual electrospray ionization (ESI) source. Source operating conditions were: $3 \mathrm{kV}$ spray voltage, $120^{\circ} \mathrm{C}$ ion source temperature, $200^{\circ} \mathrm{C}$ desolvation temperature, $200 \mathrm{~L} / \mathrm{h}$ desolvation gas flow rate, and $50 \mathrm{~L} / \mathrm{h}$ cone gas flow rate. Mass spectra were acquired in the positive-ion centroid mode with internal lock mass correction by scanning from 100 to $600 \mathrm{~m} / \mathrm{z}$ for the accurate mass measurements of testosterone acetate and estradiol benzoate and from 100 to $1000 \mathrm{~m} / \mathrm{z}$ for the accurate mass measurements of tylosin. The lock mass used for TOF mass correction was the $[\mathrm{M}+\mathrm{H}]^{+}$ion (attenuated lock mass) and ${ }^{13} \mathrm{C}$ isotope $[\mathrm{M}+\mathrm{H}]^{+}$ion of leucine enkephalin $(1 \mathrm{ng} / \mu \mathrm{L}$ in water/acetonitrile 80:20 $\mathrm{vol} / \mathrm{vol}$, delivered to the ESI source at $10 \mu \mathrm{L} / \mathrm{min}$ using an HPLC pump). The analyte-to-reference scan ratio was 9:1. Experiments were performed at spectral acquisition time of $0.25 \mathrm{~s}$ and $1.0 \mathrm{~s}$, and the interscan time was $0.01 \mathrm{~s}$. The mass resolving power of the TOFMS (determined from the $[\mathrm{M}+\mathrm{H}]^{+}$ion of leucine enkephalin at $\mathrm{m} / \mathrm{z} 556.2771$ ) was $\sim 10,000$ (FWHM). Dynamic range enhancement was switched on. The instrument was calibrated $\sim 1 \mathrm{~h}$ (testosterone acetate) to $\sim 4 \mathrm{~h}$ (tylosin) before the infusion experiments by using a sodium formate solution. The $\mathrm{m} / \mathrm{z}$ axis was calibrated by fitting the measured reference peak mass centroids to the known reference $m / z$ values using a fifth-order polynomial equation. LTQ Orbitrap MS infusion experiments were conducted using a standard ESI source operating in positive ionization mode. Source operating conditions were: $3.5 \mathrm{kV}$ spray voltage, $275^{\circ} \mathrm{C}$ heated capillary temperature, sheath and auxiliary gas at 1.4 and 3.2 $\mathrm{L} / \mathrm{min}$, respectively. All mass spectra were acquired in profile mode using the Orbitrap mass analyzer at a mass resolving power of 7500 (FWHM, at $m / z 400 ; \sim 0.2$ s scan cycle time) and $60,000(\sim 1 \mathrm{~s}$ scan cycle time). Accurate mass measurement of the $[\mathrm{M}+\mathrm{H}]^{+}$ions of testosterone acetate and estradiol benzoate were carried out by scanning from 100 to $500 \mathrm{~m} / \mathrm{z}$, and $[\mathrm{M}+\mathrm{H}]^{+}$ions of tylosin were mass measured by scanning from 100 to $1000 \mathrm{~m} / \mathrm{z}$. Automatic gain control (AGC) of the linear ion trap was switched on. The AGC target value was 30,000 accumulated ions. The Orbitrap was calibrated $\sim 1 \mathrm{~h}$ (testosterone acetate) to $\sim 4 \mathrm{~h}$ (tylosin) before the infusion experiments by using a mixture of caffeine,
MRFA peptide and Ultramark 1600. Data were acquired in the external calibration mode.

\section{Ultra Performance Liquid Chromatography-Mass Spectrometry}

All UPLC-MS analyses were carried out by using a Waters Acquity UPLC system coupled to either the Waters TOFMS or the ThermoFisher Scientific LTQ Orbitrap XL MS. UPLC-MS/MS were performed by using a Waters Quattro Premier triple quadrupole MS. Chromatographic separation of steroid esters and coccidiostats was conducted by using a Waters Acquity BEH $\mathrm{C}_{18}$ UPLC column $(100 \times 2.1 \mathrm{~mm}$ i.d., $1.7 \mu \mathrm{m})$ thermostatted at $40{ }^{\circ} \mathrm{C}$. The injection volume was $20 \mu \mathrm{L}$. For the steroid esters, Solvent A consisted of $2 \%$ formic acid in water/methanol/acetonitrile (30:35:35 vol/ vol/ vol) and Solvent B of $2 \%$ formic acid in methanol/ acetonitrile (50:50 vol/vol). Gradient elution was linearly programmed as follows: $0 \mathrm{~min} 0 \% \mathrm{~B}, 0.34 \mathrm{~min} 0 \%$ $\mathrm{B}, 4 \mathrm{~min} 100 \% \mathrm{~B}, 5 \mathrm{~min} 100 \% \mathrm{~B}, 5.06 \mathrm{~min}$ 0\% B, $6.66 \mathrm{~min}$ $0 \% \mathrm{~B}$, at a flow rate of $0.6 \mathrm{~mL} / \mathrm{min}$. The coccidiostats were separated by using a gradient of $10 \mathrm{mM}$ ammonium formate in water (Solvent $\mathrm{A}$ ) and $10 \mathrm{mM}$ ammonium formate in water/methanol (10:90 vol/ vol) (Solvent $B$ ), at a flow rate of $0.5 \mathrm{~mL} / \mathrm{min}$. That gradient was linearly programmed as follows: $0 \mathrm{~min} 10 \% \mathrm{~B}, 1.34 \mathrm{~min}$ $10 \%$ B, 2.66 min $50 \%$ B, $4.44 \mathrm{~min} 100 \%$ B, $5.34 \mathrm{~min} 100 \%$ B, 5.36 10\% B, 6.22 min 10\% B. Post-column splitting of the UPLC effluent was applied, resulting in a flow rate of $0.2 \mathrm{~mL} / \mathrm{min}$ into the ion source of the MS instruments.

Different from the infusion experiments, the TOFMS ESI reference and sample sprays were at $2.5 \mathrm{kV}$, the desolvation temperature at $375{ }^{\circ} \mathrm{C}$ and the desolvation gas flow rate at $500 \mathrm{~L} / \mathrm{h}$. Full-scan data were acquired from 100 to $600 \mathrm{~m} / \mathrm{z}$ at an acquisition rate of $0.25 \mathrm{~s}$ per spectrum throughout the UPLC-TOFMS experiments. LTQ Orbitrap MS settings were changed to sheath and auxiliary gases set at 1.8 and $5.0 \mathrm{~L} / \mathrm{min}$ and heated capillary temperature at $320^{\circ} \mathrm{C}$ when operated in the UPLC-MS mode. Mass spectra were acquired in profile mode from 100 to $500 \mathrm{~m} / \mathrm{z}$. External calibration of the mass analyzers was performed daily before starting the analyses.

Additional steroid esters in hair analyses were performed by UPLC-QqQ-MS/MS with the mass spectrometer operated in the MRM mode by using the same UPLC column and mobile phase composition. The gradient was modified to: $0 \mathrm{~min} 0 \% \mathrm{~B}, 0.50 \mathrm{~min} 0 \% \mathrm{~B}, 6$ $\min 100 \% \mathrm{~B}, 7.5 \mathrm{~min} 100 \% \mathrm{~B}, 7.6 \mathrm{~min} 0 \% \mathrm{~B}, 10 \mathrm{~min} 0 \%$ $\mathrm{B}$, at a flow rate of $0.4 \mathrm{~mL} / \mathrm{min}$. The injection volume was $40 \mu \mathrm{L}$. The mass spectrometer was operated by using the ion source and acquisition parameters as described in reference [18], with the following modifications: instead of two, three MRM transitions were acquired for each analyte. Furthermore, different colli- 
Table 2. Summary of results from 50 mass measurements for $[\mathrm{M}+\mathrm{H}]^{+}$ions of testosterone acetate, estradiol benzoate, and tylosin by using TOFMS at a mass resolving power of 10,000 and scan duration of 0.25 and $1 \mathrm{~s}$. The mean of the measured mass, mass error, and $95 \%$ confidence limit are shown

\begin{tabular}{|c|c|c|c|c|c|c|c|}
\hline \multirow[t]{2}{*}{ Compound } & \multirow[t]{2}{*}{$\begin{array}{c}\text { Theoretical } m / z \text { of } \\
{[\mathrm{M}+\mathrm{H}]^{+} \text {ion }}\end{array}$} & \multicolumn{2}{|c|}{$\begin{array}{l}\text { Mean measured } m / z \\
\quad(n=50)\end{array}$} & \multicolumn{2}{|c|}{$\begin{array}{l}\text { Mass error } \\
{[\mathrm{ppm}]^{\mathrm{a}}}\end{array}$} & \multicolumn{2}{|c|}{$\begin{array}{c}\lambda_{\text {ppm }}(95 \%) \\
{[\mathrm{ppm}]^{\mathrm{b}}}\end{array}$} \\
\hline & & $0.25 \mathrm{~s} / \mathrm{scan}$ & 1 s/scan & $0.25 \mathrm{~s} / \mathrm{scan}$ & 1 s/scan & $0.25 \mathrm{~s} / \mathrm{scan}$ & 1 s/scan \\
\hline Testosterone acetate & 331.2267 & 331.2275 & 331.2276 & 2.2 & 2.9 & 7.7 & 3.2 \\
\hline Estradiol benzoate & 377.2111 & 377.2106 & 377.2117 & -1.5 & 1.5 & 3.7 & 7.6 \\
\hline Tylosin & 916.5264 & 916.5272 & 916.5272 & 0.80 & 0.81 & 4.8 & 5.2 \\
\hline
\end{tabular}

${ }^{a} 10^{6} \times[($ mean measured $\mathrm{m} / \mathrm{z}$-theoretical $\mathrm{m} / \mathrm{z}) /$ theoretical $\mathrm{m} / \mathrm{z}]$.

bThe $95 \%$ confidence limit, $1.96 \times$ standard deviation.

sion energies, ranging from 5 to $45 \mathrm{eV}$, were used for each MRM transition.

\section{Results and Discussion}

\section{General Considerations of Precision and Accuracy of Mass Measurement}

Provided the determination of exact masses is not impaired by isobaric interferences, the error in a measured mass is composed of two components: the systematic error (accuracy) and the statistical or random error (the precision of the measurement). The mass measurement precision and accuracy should be taken into consideration for the establishment of the smallest possible mass tolerance window that can be used to plot selective RICs. The precision and accuracy of a mass measurement can be assessed by making multiple independent measurements at the mass of interest and performing a statistical analysis of the data. Infusion mass measurement experiments were carried out by using the TOFMS and the LTQ Orbitrap MS, and $1 \mathrm{ng} / \mu \mathrm{L}$ standard solutions of testosterone acetate, estradiol benzoate, and tylosin infused at $3 \mu \mathrm{L} / \mathrm{min}$. The mass assigned to the $[\mathrm{M}+\mathrm{H}]^{+}$ions of the analytes in each acquired mass spectrum was treated as an independent measurement. Fifty mass spectra of each analyte were selected to provide two sets of mass measurement data acquired at different Orbitrap mass resolving powers $(7500$ versus 60,000$)$ and different TOF scan cycle times ( $0.25 \mathrm{~s}$ versus $1 \mathrm{~s})$. The results using TOFMS in the internal calibration mode are presented in Table 2. Results from identical analyses but using the LTQ Orbitrap MS at a mass resolving power of 7500 and 60,000 are given in Table 3 . The mean values of the mass measurements were all within $3 \mathrm{ppm}$ of their theoretical $\mathrm{m} / \mathrm{z}$ values. The precision of the mass measurement was determined by calculating the $95 \%$ confidence limit $\left(\lambda_{\mathrm{ppm}}\right)$, assuming a normal distribution. The $95 \%$ confidence limit of mass measurement precision for TOFMS ranged from 3.2 to $7.7 \mathrm{ppm}$, in accordance with the results found by Blom [19], and from 0.58 to $1.4 \mathrm{ppm}$ when the LTQ Orbitrap MS was used. More general considerations about mass measurement accuracy and precision can be found in the Supplemental section. Both instruments were capable of providing a mass measurement accuracy of $<3 \mathrm{ppm}$, provided the signals being measured were composed of ions with a single elemental composition at the resolving power provided by the mass analyzer. A remarkable improvement of mass measurement precision was achieved with LTQ Orbitrap MS compared with TOFMS. The results clearly suggested that the random error dominated the total error in a mass measurement performed by this TOFMS, while systematic and random error contributed equally to the total error in a mass measurement carried out with LTQ Orbitrap MS. Therefore, the latter instrument allowed the use of RICs having very narrow mass tolerance windows ( $\leq 5 \mathrm{ppm}$ ) as long as the mass measurement was not impaired by interferences. Our TOFMS mass measurements were less precise and RIC mass tolerance windows of least 10 ppm were used.

Table 3. Summary of results from 50 mass measurements for $[\mathrm{M}+\mathrm{H}]^{+}$ions of testosterone acetate, estradiol benzoate, and tylosin by using Orbitrap MS at a mass resolving power of 7500 and 60,000 . The mean of the measured mass, mass error, and $95 \%$ confidence limit are shown

\begin{tabular}{|c|c|c|c|c|c|c|c|}
\hline \multirow[b]{2}{*}{ Compound } & \multirow{2}{*}{$\begin{array}{c}\text { Theoretical } m / z \text { of } \\
{[\mathrm{M}+\mathrm{H}]^{+} \text {ion }} \\
\end{array}$} & \multicolumn{2}{|c|}{$\begin{array}{l}\text { Mean measured } m / z \\
(n=50)\end{array}$} & \multicolumn{2}{|c|}{ Mass error $[\mathrm{ppm}]^{\mathrm{a}}$} & \multicolumn{2}{|c|}{$\lambda_{\mathrm{ppm}}(95 \%)[\mathrm{ppm}]^{\mathrm{b}}$} \\
\hline & & $R=7500$ & $\mathrm{R}=60,000$ & $\mathrm{R}=7500$ & $R=60,000$ & $R=7500$ & $R=60,000$ \\
\hline Testosterone acetate & 331.2267 & 331.2275 & 331.2270 & 2.4 & 0.66 & 1.4 & 0.88 \\
\hline Estradiol benzoate & 377.2111 & 377.2115 & 377.2111 & 0.96 & -0.05 & 0.67 & 0.76 \\
\hline Tylosin & 916.5264 & 916.5261 & 916.5245 & -0.40 & -2.1 & 0.75 & 0.58 \\
\hline
\end{tabular}

${ }^{a}$ Difference between the mean of the measured mass and the theoretical mass.

bThe $95 \%$ confidence limit, 1.96 x standard deviation. 


\section{Full Scan Accurate Mass UPLC-MS of Steroid Esters in Hair Extracts}

To evaluate the performance of accurate mass full-scan MS in the detection of steroid esters, extracts of hair were fortified at four different concentration levels (Table 1). Extract spiking level B corresponded to the LC-MS/MS validated CC $\beta$ levels (detection capability according to the definition in reference [15]) of 5 to 110 $\mathrm{ng} / \mathrm{g}$ hair at $100 \%$ recovery. A significant improvement of RIC selectivity was obtained by reducing the mass tolerance window from $500 \mathrm{mDa}$ to $10 \mathrm{ppm}$, as shown for testosterone acetate analyzed by UPLC-LTQ Orbitrap MS in Figure 1. An additional reduction of matrix related signals and improvement of signal to noise ratio could be obtained by reducing the RIC window further from 10 to 5 ppm (Figure 1c). The effect of mass resolving power on RIC detection of steroid esters in hair extracts is illustrated for testosterone isocaproate in Figure 2. Testosterone isocaproate was detected in hair extract at a retention time of $3.00 \mathrm{~min}$ in the 5 ppm RIC window when the experiment was carried out at a resolving power of 60,000 (Figure 2b). However, the RIC obtained from data that were acquired at a mass resolving power of 7500 (Figure 2a) did not reveal the presence of any testosterone isocaproate in the hair extract. Insufficient sensitivity of UPLC-Orbitrap MS at
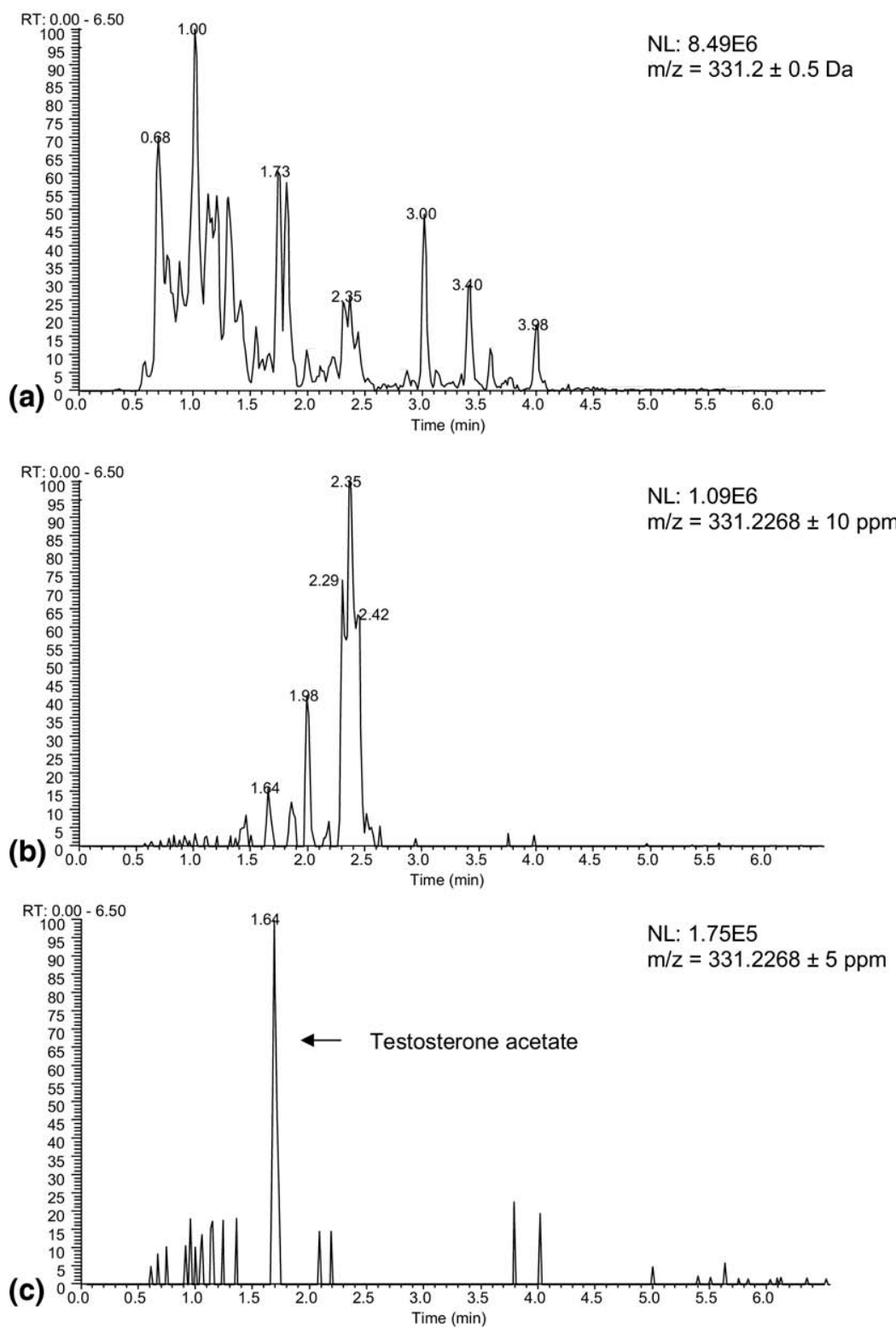

Figure 1. Selectivity enhancement by using narrow mass tolerance windows. Detection of testosterone acetate in hair extract at $5 \mathrm{ng} / \mathrm{mL}$ level using UPLC-Orbitrap MS at 60,000 resolving power. Reconstructed ion chromatograms corresponding to the theoretical $\mathrm{m} / \mathrm{z}$ of the protonated molecule of testosterone acetate $(\mathrm{m} / \mathrm{z} 331.2268)$ using mass tolerance filters of $500 \mathrm{mDa}(\mathbf{a}), 10 \mathrm{ppm}$ (b), and 5 ppm (c). 

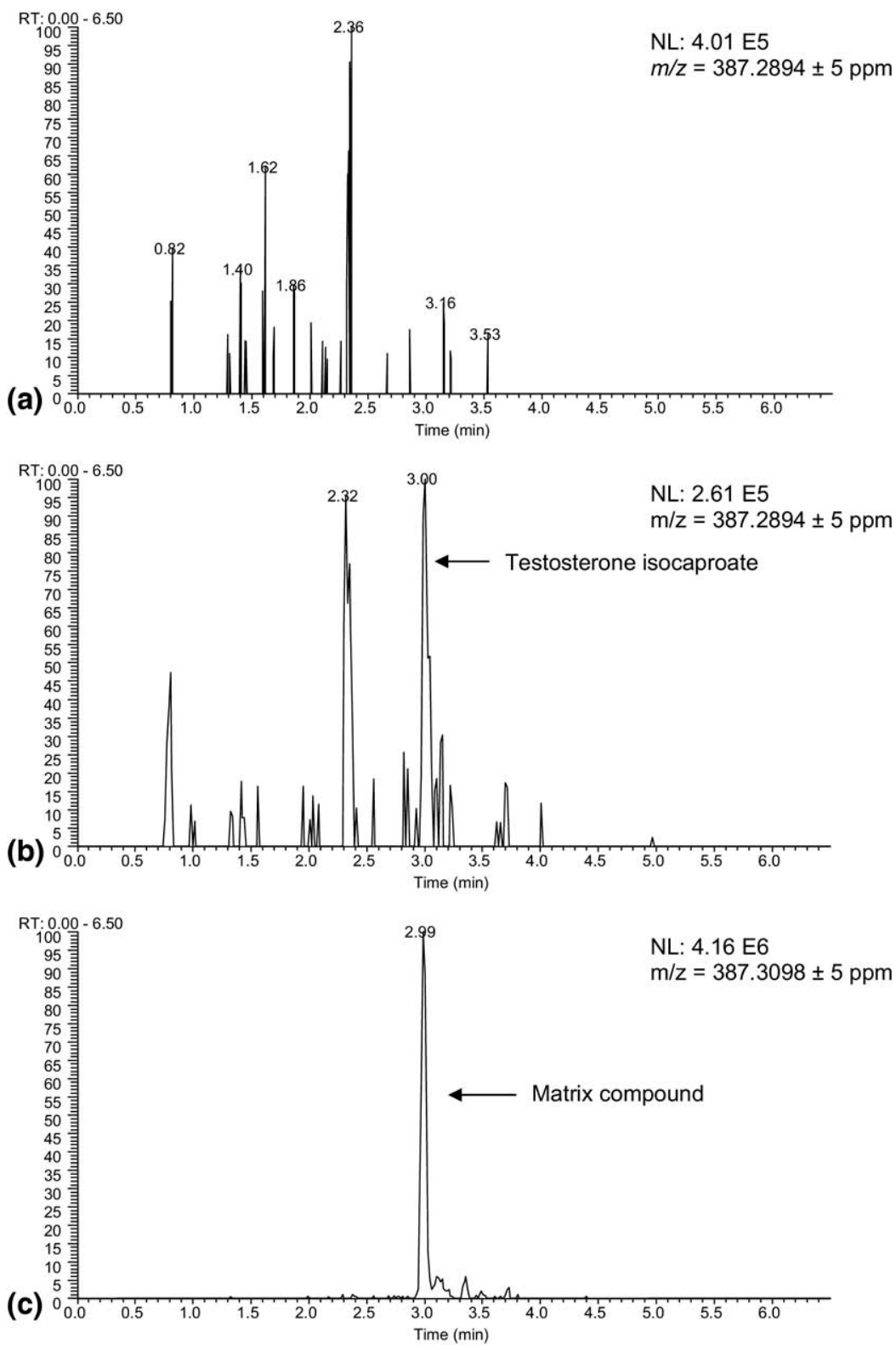

Figure 2. Discrimination of testosterone isocaproate from an isobaric co-eluting sample matrix compound required a resolving power of $\sim 37,000$. Detection of testosterone isocaproate in hair extract at $5 \mathrm{ng} / \mathrm{mL}$ level using UPLC-Orbitrap MS at 7500 (a) and 60,000 (b) and (c) resolving power. Reconstructed ion chromatograms corresponding to the theoretical $\mathrm{m} / \mathrm{z}$ of the protonated molecule of testosterone acetate $(\mathrm{m} / \mathrm{z} 387.2894)$ and of a sample matrix compound at $\mathrm{m} / \mathrm{z} 387.3098$ using a mass tolerance window of $5 \mathrm{ppm}$.

7500 was not the cause as indicated by the capability to detect the analyte in a standard solution at $5 \mathrm{ng} / \mathrm{mL}$ level (data not shown). The failure to detect testosterone isocaproate at 7500 resolving power was caused by co-elution of an isobaric matrix compound (Figure 2c). The mass spectrum acquired at the retention time of testosterone isocaproate with the Orbitrap MS operating at a resolving power of 60,000 (Figure 3a) showed the $[\mathrm{M}+\mathrm{H}]^{+}$ion of the analyte at a measured $\mathrm{m} / \mathrm{z}$ of 387.2887 (mass error $=-1.7 \mathrm{ppm}$ ) together with an isobaric ion at $\mathrm{m} / \mathrm{z}$ 387.3098. A mass resolving power of $\sim 37000$ was required to baseline separate the ion of the matrix compound from the protonated molecular ion of testosterone isocaproate. In the mass spectrum acquired at a resolving power of 7500 (Figure 3b), the ions merged into a single mass peak, with a centroid mass corresponding to a weighted arithmetic mass average. As a result, the difference between the measured $\mathrm{m} / \mathrm{z}$ of 387.3102 and the theoretical $m / z$ of the $[\mathrm{M}+\mathrm{H}]^{+}$ion of testosterone isocaproate was $50 \mathrm{ppm}$, exceeding the RIC mass tolerance window, and causing the disappearance of the analyte signal. Examination of the mass spectra acquired at the retention time of the steroid esters revealed the presence of isobaric background ions in the 

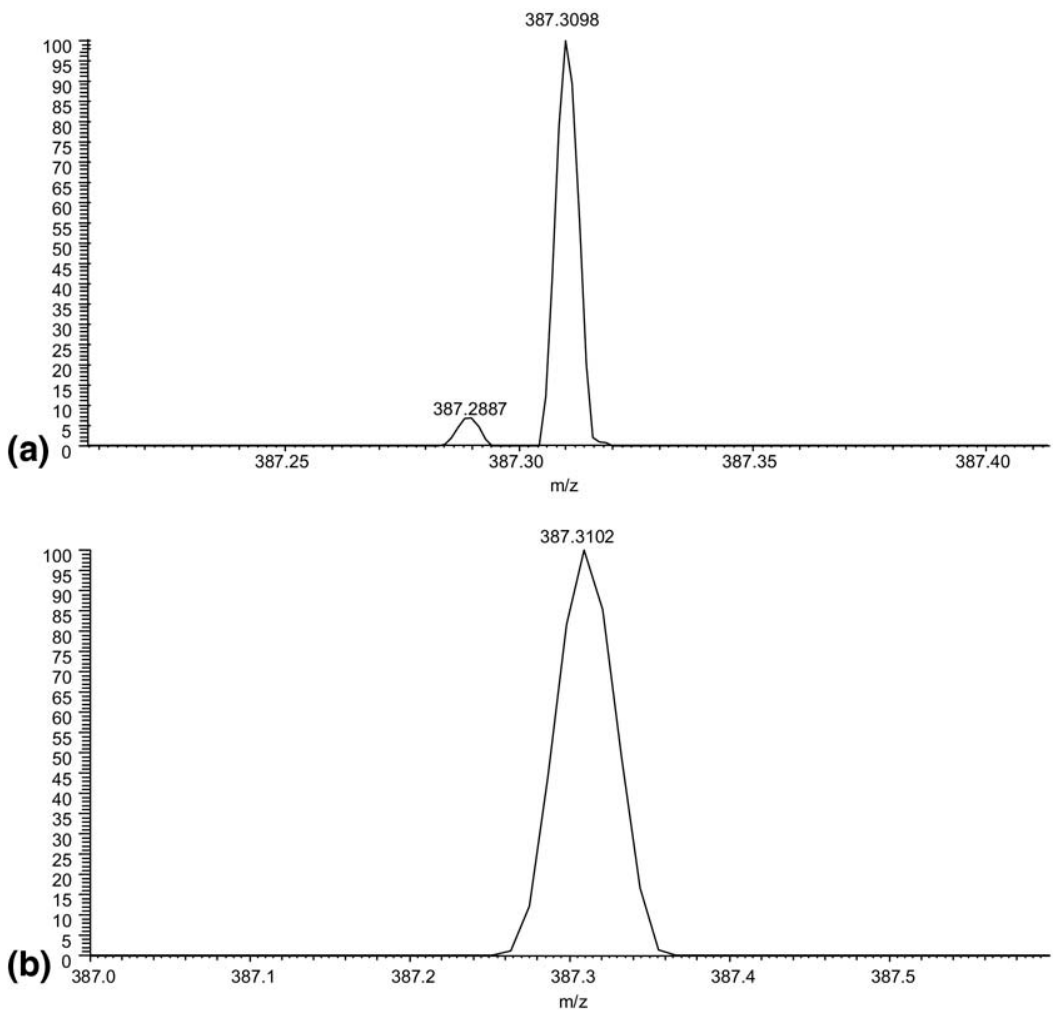

Figure 3. Full-scan Orbitrap mass spectra acquired at a resolving power of 60,000 (a) and 7500 (b). Separation of the $[\mathrm{M}+\mathrm{H}]^{+}$ion of testosterone isocaproate $(\mathrm{m} / \mathrm{z} 387.2887)$ from the ion of a matrix compound $(\mathrm{m} / \mathrm{z} 387.3098)$ at a resolving power of 60,000 . No separation between analyte ions and matrix compound ions at a resolving power of 7500 .

spectra of almost every analyte. A resolving power of 7500 was sufficient to base line separate the analyte ions of six steroid esters from background ions. However, a resolving power of 15,000 to 40,000 was necessary to completely discriminate the protonated molecular ions of the remaining steroid esters from interfering background ions. An actual resolving power of 60,000 ensured a sufficient selectivity to distinguish all molecular ions from interfering co-eluting matrix compounds and enabled the detection of all steroid esters in hair extracts in the RICs at the expected retention times, even at the lowest concentration level (Figure 4). However, a mass tolerance window of $\pm 5 \mathrm{ppm}$ revealed to be of critical importance, i.e., several steroid esters could not be detected in their $10 \mathrm{ppm}$ mass tolerance RIC, owing to insufficient selectivity.

To establish the mutual effect of matrix compounds and mass resolving power on the detection performance of full-scan UPLC-LTQ Orbitrap MS, solutions of steroid esters in pure solvent [water/acetonitrile/methanol (30:35:35)] at concentration levels equal to those in the fortified hair extracts were analyzed as well. At 60,000 mass resolving power, all steroid esters were detected in the RICs at the lowest concentration level (level A, Table 1) using a mass tolerance window of 5 ppm (data not shown). Owing to the absence of matrix interferences, steroid esters were also detected at the lowest concentration level at a mass resolving power of
7500 (data not shown), with the exception of testosterone cypionate. The detection of this compound in a 5 ppm RIC was hampered by mass interference of the sodium adduct ion of the ubiquitous contaminant dioctyl phthalate (see Supplementary Figures 1 and 2).

Figure 5 shows the MRM chromatograms obtained from the UPLC-QqQ-MS/MS analysis of hair samples fortified with steroid esters at concentration level A. The MRM method employed involved the transition of the $[\mathrm{M}+\mathrm{H}]^{+}$precursor ions to three product ions. To visualize the selectivity difference between the MRM and the full-scan approach, only the MRM transitions with the highest signal to noise ratio are shown in Figure 5. It should be noted that the analytes were spiked into the hair samples and not into the extracts as in the case of the UPLC-Orbitrap MS analyses. Thus, the MRM signals might have been influenced by a less than $100 \%$ recovery. A comparison of Figure 5 with the traces e, $\mathrm{f}, \mathrm{h}$, and $\mathrm{k}$ shown in Figure 4 revealed that the MRM method was more selective in detecting steroid esters in hair, albeit with some exceptions in particular nortestosterone phenylpropionate. This general conclusion could also be drawn on the basis of a comparison of the selectivity of the other transitions employed in the MRM method with the selectivity obtained with UPLC-Orbitrap MS at a resolving power of 60,000.

The accurate mass of the $[\mathrm{M}+\mathrm{H}]^{+}$ions of the steroid esters was obtained by averaging a number of spectra 

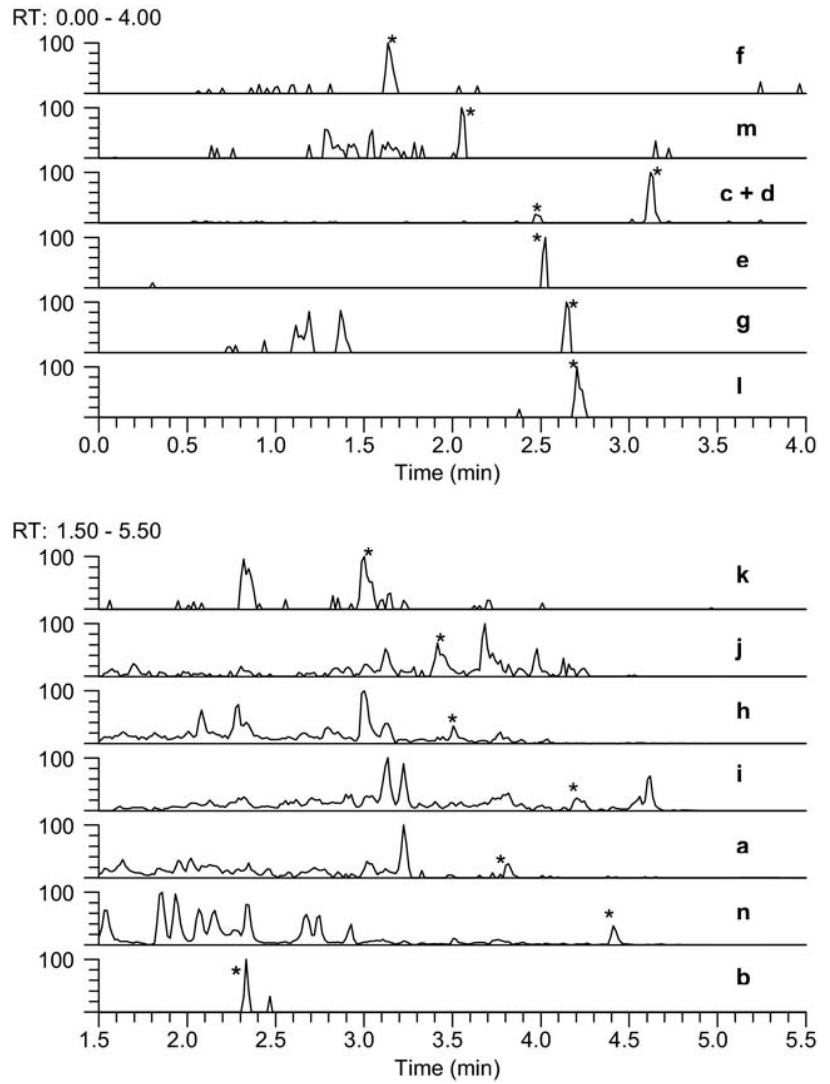

Figure 4. Detection of steroid esters in hair extracts at concentration level A using UPLC-Orbitrap MS at a resolving power of 60,000 . Reconstructed ion chromatograms corresponding to the theoretical mass of the $[\mathrm{M}+\mathrm{H}]^{+}$ions of steroid esters except trace $\mathrm{c}+\mathrm{d}$, which is the $\mathrm{m} / \mathrm{z} 255.1743$ fragment ion chromatogram of estradiol valerate and estradiol enanthate. A $\pm 5 \mathrm{ppm}$ mass tolerance was used to filter the data. The RICs of the individual steroid esters are identified by letters corresponding to those presented in Table 4. The chromatographic peaks of the steroid esters are labeled with an asterisk.

across the LC peak (at $\sim 30 \%$ of the peak height). Instead of the protonated molecular ion of estradiol enanthate and estradiol valerate, the mass of the fragment ion at theoretical $\mathrm{m} / \mathrm{z} 255.1743$ was measured because of the low ion abundance of the $[\mathrm{M}+\mathrm{H}]^{+}$ion. The utilization of a high resolving power of 60,000 to remove interferences from isobaric matrix compounds produced $<3 \mathrm{ppm}$ mass errors for all steroid esters at all concentration levels (Table 4). The root-mean-square (RMS) mass error showed a small but insignificant increase at the lowest concentration level. However, the signal to noise ratio in some of the RICs decreased to such an extent that reliable quantification became impossible. Fortunately, for a screening method aiming at detection and identification of banned growth-promoters for which no maximum residue limit (MRL) has been set, quantification is far less relevant than confirmation of identity.

A mass resolving power of 7500 proved to be inadequate for a reliable detection of steroid esters in hair extracts. At the lowest concentration level, eight of the
14 analytes were not detected owing to the failure to resolve their protonated molecular ions from co-eluting isobaric matrix compounds. Mass errors exceeding 3 ppm were obtained frequently (data are summarized in Supplementary Table 4). Decrease of the sample concentration resulted in an increase of mass errors for several compounds at 7500 resolving power.

To assess the effect of sample concentration on mass measurement accuracy of Orbitrap MS and TOFMS, a series of experiments were performed using a varying concentration of steroid esters in calibration standard solutions. The results of these experiments can be found and are discussed in the Supplemental section (Supplementary Tables 1, 2, and 3).

UPLC-TOFMS at 10,000 mass resolving power was expected to yield results similar to those obtained at the 7500 resolving power LTQ Orbitrap MS setting. The high full-scan sensitivity enabled the detection of the analytes at low $\mathrm{ng} / \mathrm{mL}$ level in solvent based standards. However, in hair extracts UPLC-TOFMS failed to detect many of the target analytes in the 10 ppm RICs: only 5 analytes were found at the lowest concentration level, versus 13 analytes in a standard solution. This showed that although the instrument's sensitivity was sufficient to detect the analytes at the required level, the provided mass resolving power of 10,000 was too low to resolve analyte ion and co-eluting isobaric compounds from the sample matrix. Obviously, SPE sample clean-up did not eliminate the problems associated with the presence of interfering compounds in the sample matrix. Also, the enhanced chromatographic resolving power provided by UPLC was not sufficient to separate interfering isobaric matrix compounds from analytes. In addition, the mass resolving power of 10,000 was not sufficient to

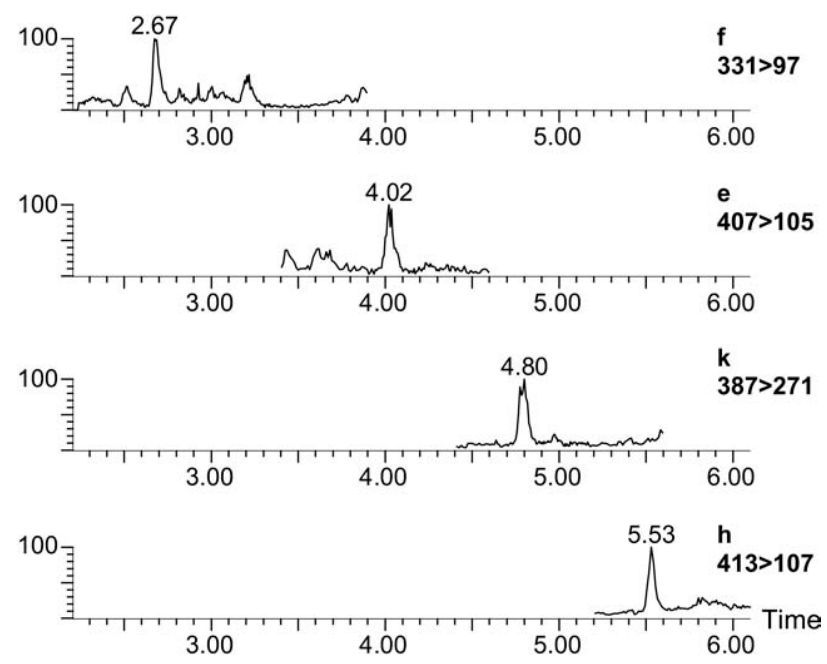

Figure 5. MRM chromatograms obtained from the UPLC-QqQMS/MS analysis of hair fortified with steroid esters at concentration level A. The traces of the individual steroid esters are identified by letters corresponding to those presented in Table 4. The $m / z$ of precursor ion and product ion are depicted on the traces. The chromatographic run time was divided in seven time windows containing four to seven preselected transitions. 
Table 4. Accurate mass measurement of steroid esters in hair extracts fortified at four concentration levels using full-scan UPLC-Orbitrap MS at a resolving power of 60,000

\begin{tabular}{|c|c|c|c|c|c|c|}
\hline & Compound & $\begin{array}{c}\text { Theoretical } \mathrm{m} / \mathrm{z} \\
{[\mathrm{M}+\mathrm{H}]^{+} \text {ion }[\mathrm{Da}]}\end{array}$ & & Mass & ppm] & \\
\hline & Concentration level $^{a}$ & & A & B & C & $\mathrm{D}$ \\
\hline$A$ & Boldenone undecylenate & 453.3363 & -2.8 & -2.3 & -2.3 & -2.3 \\
\hline $\mathrm{B}$ & Estradiol benzoate & 377.2111 & -0.56 & -1.4 & -1.4 & -1.2 \\
\hline $\mathrm{C}$ & Estradiol enanthate & $255.1743^{b}$ & -1.3 & -0.86 & -0.86 & -1.0 \\
\hline $\mathrm{D}$ & Estradiol valerate & $255.1743^{\mathrm{b}}$ & -1.1 & -1.1 & -1.1 & -0.67 \\
\hline $\mathrm{E}$ & Nortestosterone phenylpropionate & 407.2581 & 2.5 & -1.8 & -1.7 & -0.83 \\
\hline $\mathrm{F}$ & Testosterone acetate & 331.2268 & -1.7 & -1.5 & -1.3 & -1.3 \\
\hline $\mathrm{G}$ & Testosterone benzoate & 393.2424 & -1.2 & -1.8 & -1.8 & -2.0 \\
\hline $\mathrm{H}$ & Testosterone cypionate & 413.3050 & -1.6 & -1.4 & -1.4 & -1.5 \\
\hline 1 & Testosterone decanoate & 443.3520 & -2.8 & -2.6 & -2.6 & -2.8 \\
\hline $\mathrm{J}$ & Testosterone enanthate & 401.3050 & -2.4 & -2.1 & -2.1 & -2.4 \\
\hline $\mathrm{K}$ & Testosterone isocaproate & 387.2894 & -2.0 & -0.72 & -0.72 & -1.7 \\
\hline $\mathrm{L}$ & Testosterone phenylpropionate & 421.2737 & -1.8 & -2.1 & -2.1 & -1.8 \\
\hline $\mathrm{M}$ & Testosterone propionate & 345.2424 & -2.6 & -1.6 & -1.3 & -1.5 \\
\hline $\mathrm{N}$ & Testosterone undecanoate & 457.3676 & -2.9 & -2.8 & -2.8 & -2.7 \\
\hline & RMS [ppm] & & 2.1 & 1.8 & 1.8 & 1.8 \\
\hline
\end{tabular}

${ }^{a}$ Concentration levels are specified in Table 1.

${ }^{\mathrm{b}}$ Fragment ion.

generate mass measurement data of adequate accuracy in the presence of a complex matrix background. As shown in Supplementary Table 5, the mass measurement results frequently differed more than $5 \mathrm{ppm}$ from the expected value owing to interference of co-eluting isobaric compounds originating from the hair matrix. These results were consistent with those obtained using Orbitrap MS at 7500 resolving power.

All results have been summarized in Table 5; for each steroid ester the number of false compliant (false negative) results, i.e., the number of concentration levels at which the analyte was not detected, and the average mass error are presented.

\section{Full Scan Accurate Mass UPLC-MS of Coccidiostats in Feed Extracts}

With the exception of amprolium, all coccidiostats, carbadox, and olaquindox were detected at the lowest concentration level of $15 \mathrm{ng} / \mathrm{mL}$ (corresponding with $240 \mathrm{ng} / \mathrm{g}$ feed at $100 \%$ recovery) by using full-scan UPLC-LTQ Orbitrap MS at a mass resolving power of 60,000. Detection was accomplished by RICs using a mass tolerance window of $5 \mathrm{ppm}$, but this appeared to be less critical; a window of 10 ppm performed equally well. Also, the mass resolving power was less critical versus the steroid case; UPLC-TOFMS enabled the

Table 5. Number of false compliant (false negative) results and average mass measurement accuracy (absolute values) obtained for steroid esters in hair extracts using UPLC-TOFMS at 10,000 resolving power and UPLC-Orbitrap MS at 7500 and 60,000 resolving power

\begin{tabular}{|c|c|c|c|c|c|c|}
\hline \multirow[b]{2}{*}{ Compound } & \multicolumn{3}{|c|}{$\mathrm{N}^{\mathrm{a}}$} & \multicolumn{3}{|c|}{ Mass error ${ }^{b}[p p m]$} \\
\hline & $\mathrm{R}=7500$ & $R=10,000$ & $\mathrm{R}=60,000$ & $\mathrm{R}=7500$ & $R=10,000$ & $R=60,000$ \\
\hline Boldenone undecylenate & 0 & 1 & 0 & 4.6 & 7.6 & 2.4 \\
\hline Estradiol benzoate & 1 & 3 & 0 & 0.7 & & 1.1 \\
\hline Estradiol enanthate & 0 & 0 & 0 & 0.5 & 4.4 & 1.0 \\
\hline Estradiol valerate & 0 & 0 & 0 & 0.8 & 2.9 & 1.0 \\
\hline Nortestosterone phenylpropionate & 1 & 1 & 0 & 1.5 & 4.3 & 1.7 \\
\hline Testosterone acetate & 1 & 1 & 0 & 0.6 & 1.8 & 1.4 \\
\hline Testosterone benzoate & 0 & 4 & 0 & 1.6 & & 1.7 \\
\hline Testosterone cypionate & 1 & 4 & 0 & 3.8 & & 1.5 \\
\hline Testosterone decanoate & 0 & 1 & 0 & 5.2 & 11 & 2.7 \\
\hline Testosterone enanthate & 2 & 2 & 0 & 6.2 & 6.8 & 2.3 \\
\hline Testosterone isocaproate & 4 & 3 & 0 & & & 1.3 \\
\hline Testosterone phenylpropionate & 3 & 0 & 0 & & 3.9 & 2.0 \\
\hline Testosterone propionate & 1 & 0 & 0 & 2.7 & 5.1 & 1.7 \\
\hline Testosterone undecanoate & 0 & 0 & 0 & 1.8 & 2.6 & 2.8 \\
\hline
\end{tabular}

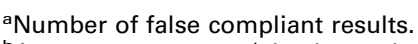

${ }^{b}$ Average mass error (absolute values) calculated form the mass errors obtained at those concentration levels on which the analyte was detected. The average mass error was calculated for those analytes that were detected at a minimum of two concentration levels. 

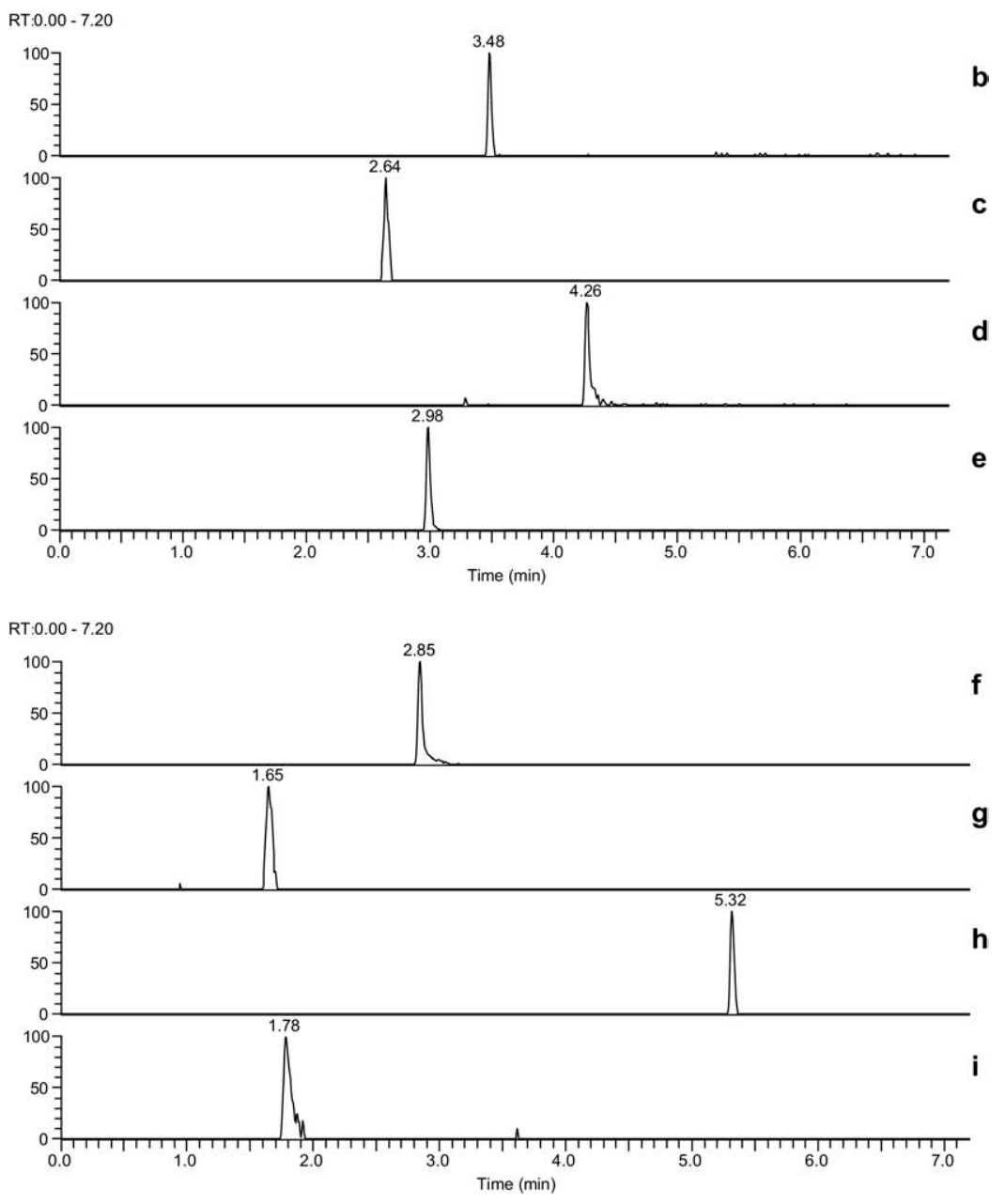

Figure 6. Detection of coccidiostats in feed extracts at a concentration of $15 \mathrm{ng} / \mathrm{mL}$ using UPLC-Orbitrap MS at a resolving power of 7500. Extracted ion chromatograms corresponding to the theoretical mass of the $[\mathrm{M}+\mathrm{H}]^{+}$ions of target analytes. A \pm 5 ppm mass tolerance was used to filter the data. The RICs of the individual coccidiostats are identified by letters corresponding to those presented in Table 6. Amprolium was not detected.

detection of all coccidiostats, whereas UPLC-Orbitrap MS at a resolving power of 7500 only missed the detection of amprolium at the lowest concentration level (Figure 6). The successful detection of coccidiostats in feed extracts in contrast to the failure to detect steroid esters in hair extracts at a mass resolving power of 7500 was most likely explained by the difference in relative concentration level of compounds versus the sample matrix. The procedure for the analysis of steroid esters yielded an extract containing the equivalent of $1 \mathrm{~g}$ of hair sample per $\mathrm{mL}$ versus $0.06 \mathrm{~g}$ of feed sample per $\mathrm{mL}$.

The majority of the analytes had mass accuracies $<5$ ppm by using Orbitrap MS and $<10$ ppm by using TOFMS. Table 6 shows a summary of the results. Individual mass errors obtained at each concentration level by using Orbitrap and TOFMS are presented in Supplementary Tables 6, 7, and 8. The resolving power did not have a significant influence on the average mass errors. This indicated that a medium resolving power of
7500 to 10,000 was sufficient to prevent inaccurate mass assignment owing to matrix interferences. The exception was the mass measurement accuracy of dimetridazole which was adversely affected by co-elution of a matrix compound having a ${ }^{13} \mathrm{C}$ isotope $[\mathrm{M}+\mathrm{H}]^{+}$ion unresolved from the protonated molecular ion of dimetridazole. Owing to composition diversity between feed batches, the interfering matrix compound was present in the feed extract used for UPLC-TOFMS, but not in UPLC- LTQ Orbitrap MS analyses.

\section{Conclusions}

The mass resolving power required to prevent false compliant (false negative) results in residue screening methods based on full-scan accurate mass LC-MS is dependent on many factors, such as analyte concentration, matrix, and sample preparation procedure. This study demonstrates that the mass resolving power determines the true utility of full-scan accurate mass 
Table 6. Average mass measurement accuracy (absolute values) obtained for coccidiostats in animal feed extracts using UPLC-TOFMS at 10,000 resolving power and UPLC-Orbitrap MS at 7500 and 60,000 resolving power

\begin{tabular}{|c|c|c|c|c|c|c|}
\hline & \multirow[b]{2}{*}{ Compound } & \multirow{2}{*}{$\begin{array}{l}\text { Chemical formula of } \\
\qquad[\mathrm{M}+\mathrm{H}]^{+} \text {ion }\end{array}$} & \multirow{2}{*}{$\begin{array}{c}\text { Theoretical } m / z \\
{[\mathrm{M}+\mathrm{H}]^{+} \text {ion }[\mathrm{Da}]}\end{array}$} & \multicolumn{3}{|c|}{ Average mass error ${ }^{a}[p p m]$} \\
\hline & & & & $R=7500$ & $R=10,000$ & $\mathrm{R}=60,000$ \\
\hline $\bar{a}$ & Amprolium & $\mathrm{C}_{14} \mathrm{H}_{19} \mathrm{~N}_{4}$ & 243.1604 & 3.9 & 3.9 & 4.7 \\
\hline$b$ & Carbadox & $\mathrm{C}_{11} \mathrm{H}_{11} \mathrm{~N}_{4} \mathrm{O}_{4}$ & 263.0775 & 2.2 & 4.1 & 2.9 \\
\hline c & Dimetridazole & $\mathrm{C}_{5} \mathrm{H}_{8} \mathrm{~N}_{3} \mathrm{O}_{2}$ & 142.0611 & 3.4 & 17 & 5.0 \\
\hline d & Ethopabate & $\mathrm{C}_{12} \mathrm{H}_{16} \mathrm{NO}_{4}$ & 238.1074 & 1.9 & 1.3 & 2.1 \\
\hline $\mathrm{e}$ & Furazolidone & $\mathrm{C}_{8} \mathrm{H}_{8} \mathrm{~N}_{3} \mathrm{O}_{5}$ & 226.0459 & 3.6 & 2.3 & 4.0 \\
\hline$f$ & Meticlorpindol & $\mathrm{C}_{7} \mathrm{H}_{8} \mathrm{Cl}_{2} \mathrm{NO}$ & 191.9978 & 4.0 & 4.2 & 4.3 \\
\hline $\mathrm{g}$ & Olaquindox & $\mathrm{C}_{12} \mathrm{H}_{14} \mathrm{~N}_{3} \mathrm{O}_{4}$ & 264.0979 & 4.8 & 4.7 & 4.6 \\
\hline $\mathrm{h}$ & Robenidine & $\mathrm{C}_{15} \mathrm{H}_{14} \mathrm{Cl}_{2} \mathrm{~N}_{5}$ & 334.0621 & 1.0 & 1.6 & 2.3 \\
\hline i & Ronidazole & $\mathrm{C}_{6} \mathrm{H}_{9} \mathrm{~N}_{4} \mathrm{O}_{4}$ & 201.0618 & 3.5 & 3.4 & 5.1 \\
\hline
\end{tabular}

${ }^{a}$ Average mass error (absolute values) calculated form the individual mass errors obtained at four concentration levels.

UPLC-MS for the analysis of banned steroid esters in hair at low ng/g level. On the other hand, for some screening applications, such as coccidiostats in animal feed at $240 \mathrm{ng} / \mathrm{g}$ level, the resolving power of the mass analyzer could have no effect at all on the capability to detect residues. Therefore, the establishment of guidelines for acceptable mass resolving power limits to avoid false compliant screening results is not useful. This study shows that the mass resolving power requirement of 20,000 (FWHM) in decision 2002/657/EC is not sufficient to enable the reliable screening of banned steroid esters in hair by using full-scan UPLC-MS.

The Orbitrap mass analyzer shows an insignificant trade-off in sensitivity for resolving power. Therefore, we would recommend using the Orbitrap at a resolving power of 60,000 in residue analysis, unless the relative slow scan cycle time of $1 \mathrm{~s}$ per scan is not compatible with the UPLC peak widths of the analytes. In this study, when the Orbitrap was used at a resolving power of 60,000 , the number of spectra acquired across many analyte UPLC peaks was only five. Therefore, the loss of analyte signal in just one spectrum would already have dramatic consequences with respect to the accuracy of the peak area. Fortunately, the stable mass measurement accuracy and high precision of the Orbitrap analyzer allowed the use of 5 ppm RICs and only a partial loss of analyte signal occurred due to the low number of spectra acquired over the chromatographic peak.

The use of appropriate mass tolerance windows is essential for reliable RICs and detection of hormone and veterinary drug residues. Mass measurement precision and accuracy as well as the resolving power provided by the instrument should be taken into account to avoid the risk of false compliant findings due to the use of too narrow mass windows. Standard addition to individual samples with realistic analyte concentrations will be the most appropriate approach for the assessment of mass tolerance windows and to monitor for any false compliant screening results.

A comparison of the results of the steroid esters analyses obtained with UPLC-Orbitrap MS at 60,000 resolving power with those of an MRM UPLC-QqQ-
MS/MS confirmatory method showed that the MRM method was more selective and sensitive for the majority of the analytes. Due to advances in the field of triple quadrupole and quadrupole ion trap technology, the new generation of tandem MS instruments enable up to hundreds of MRM transitions to be conducted in a single LC-MS/MS run. However, the targeted analysis approach has the inevitable disadvantage of being blind for non-target analytes. Therefore, for a screening method aiming at the detection of both target analytes and non-"a priori" selected compounds, such as the screening of designer anabolic steroids, the full-scan approach, using high resolving power mass analyzers, would be the method of choice.

\section{Acknowledgments}

The authors acknowledge Dr. Markus Kellmann and Richard Zuiderent from ThermoFisher for providing access to and assistance with the LTQ-Orbitrap MS measurements. The authors acknowledge Paul Zomer from RIKILT for stimulating discussions. This project was financially supported by the Dutch Ministry of Agriculture, Nature, and Food Quality.

\section{References}

1. Stolker, A. A. M.; Zuidema, T.; Nielen, M. W. F. Residue Analysis of Veterinary Drugs and Growth-promoting Agents. Trends. Anal. Chem. 2007, 26, 967-979.

2. Nielen, M. W. F.; Lasaroms, J. J. P.; Essers, M. L.; Sanders, M. B.; Oosterink, J. E.; Meijer, T.; Stolker, A. A. M. Multiresidue Analysis of $\beta$-Agonists in Bovine and Porcine Urine, Feed, and Hair Using Liquid Chromatography Electrospray Ionization Tandem Mass Spectrometry Anal. Bioanal. Chem. 2008, 391, 199-210.

3. Kaufmann, A.; Butcher, P.; Maden, K.; Widmer, M. Ultra-Performance Chromatography Coupled to Time of Flight Mass Spectrometry (UPLCTOF): A Novel Tool for Multiresidue Screening of Veterinary Drugs in Urine. Anal. Chim. Acta 2007, 586, 13-21.

4. Laks, S.; Pelander, A.; Vuori, E.; Ali-Tolppa, E.; Sippola, E.; Ojanperä, I. Analysis of Street Drugs in Seized Material without Primary Reference Standards. Anal. Chem. 2004, 76, 7375-7379.

5. Kolmonen, M.; Leinonen, A.; Pelander, A.; Ojanperä, I.A General Screening Method for Doping Agents in Human Urine by Solid Phase Extraction and Liquid Chromatography/Time-of-Flight Mass Spectrometry. Anal. Chim. Acta 2007, 585, 94-102.

6. Garcia-Reyes, J. F.; Hernando, M. D.; Ferrer, C.; Molina-Diaz, A.; Fernández-Alba, A. R. Large Scale Pesticide Multiresidue Methods in Food Combining Liquid Chromatography-Time-of-Flight Mass Spectrometry and Tandem Mass Spectrometry. Anal. Chem. 2007, 79, 7308 7323.

7. Hernando, M. D.; Mezcua, M.; Suárez-Barcena, J. M.; Fernández-Alba, A. R. Liquid Chromatography with Time-of-Flight Mass Spectrometry 
for Simultaneous Determination of Chemotherapeutant Residues in Salmon. Anal. Chim. Acta 2006, 562, 176-184.

8. Ojanperä, S.; Pelander, A.; Pelzing, M.; Krebs, I.; Vuori, E.; Ojanperä, I Isotopic Pattern and Accurate Mass Determination in Urine Drug Screening by Liquid Chromatography/Time-of-Flight Mass Spectrometry. Rapid Commun. Mass Spectrom. 2006, 20, 1161-1167.

9. Makarov, A. Denisov, E. Lange, O. Kholomeev, A. Horning S. Proceedings of the 53rd ASMS Conference on Mass Spectrometry and Allied Topics; San Antonia, TX, June, 2005.

10. Makarov, A.; Denisov, E.; Kholomeev, A.; Balschun, W.; Lange, O.; Strupat, K.; Horning, S. Performance Evaluation of a Hybrid Linear Ion Trap/Orbitrap Mass Spectrometer. Anal. Chem. 2006, 78, 2113-2120.

11. Virus, E.; Sobolevsky, T.; Rodchenkov, G. Introduction of HPLC/ Orbitrap Mass Spectrometry as Screening Method for Doping Control. J. Mass Spectrom. 2008, 43, 949-957.

12. Krauss, M.; Hollender, J. Analysis of Nitrosamines in Wastewater: Exploring the Trace Level Quantification Capabilities of a Hybrid Linear Ion Trap/Orbitrap Mass Spectrometer. Anal. Chem. 2008, 80, 834-842.

13. Makarov, A.; Denisov, E.; Lange, O.; Horning, S. Dynamic Range of Mass Accuracy in LTQ Orbitrap Hybrid Mass Spectrometer. J. Am. Soc. Mass Spectrom. 2006, 17, 977-982.

14. Nielen, M. W. F.; Engelen, M. C. van; Zuiderent, R.; Ramaker, R. Screening and Confirmation Criteria for Hormone Residue Analysis
Using Liquid Chromatography Accurate Mass Time-of-Flight, Fourier Transform Ion Cyclotron Resonance and Orbitrap Mass Spectrometry Techniques. Anal. Chim. Acta 2007, 586, 122-129.

15. Commission Decision 2002/657/EC, Official J. Eur. Commun. 2002, L221-8.

16. Calbiani, F.; Careri, M.; Elviri, L.; Mangia, A.; Zagnoni, I. Matrix Effects on Accurate Mass Measurements of Low-Molecular Weight Compounds Using Liquid Chromatography-Electrospray-Quadrupole Time-of-Flight Mass Spectrometry. J. Mass Spectrom. 2006, 41, 289-294.

17. Kaufmann, A.; Butcher, P. Strategies to Avoid False Negative Findings in Residue Analysis Using Liquid Chromatography Coupled to Timeof-Flight Mass Spectrometry. Rapid Commun. Mass Spectrom. 2006, 20 , 3566-3572.

18. Nielen, M. W. F.; Lasaroms, J. J. P.; Mulder, P. P. J.; Hende, J. van; Rhijn, J. A. van; Groot, M. J. Multiresidue Screening of Intact Testosterone Esters and Boldenone Undecylenate in Bovine Hair Using Liquid Chromatography Electrospray Tandem Mass Spectrometry. J. Chromatogr. B 2006, 830, 126-134.

19. Blom, K. F. Estimating the Precision of Exact Mass Measurements on a Orthogonal Time-of-Flight Mass Spectrometer. Anal. Chem. 2001, 73 715-719. 